\title{
A COMPARISON OF TWO MODES OF ORDER CONVERGENCE
}

\section{J. C. MATHEWS AND R. F. ANDERSON}

1. Introduction. E. S. Wolk [9] has discussed two modes of convergence of nets in partially ordered sets (in our terminology these are 2-convergence and 3-convergence). In particular, he gave a partial answer to the following question: What is a necessary and sufficient condition on a partially ordered set that these two modes of convergence be equivalent? In this paper we discuss 1-convergence and 2-convergence, asking the same question.

2. Definitions. By a partially ordered set (poset) we mean a set $S$ with a transitive, reflexive relation $\leq$ such that if $x \leq y$ and $y \leq x$, then $x=y$. We write $x<y$ iff $x \leq y$ and $x \neq y$. A subset $B$ of $S$ is called updirected (down-directed) iff for $a, b \in B$ there is $c \in B$ such that $a \leq c$ and $b \leq c(c \leq a$ and $c \leq b)$. When we refer to a net $(f ; D, \geqq)$ in $S$, we follow Kelley [4].

We now give the two definitions of order convergence that we wish to study; we also give the definition of 3-convergence appearing in Wolk.

Definition 1. A net $(f ; D, \geqq)$ in a poset $(S, \leq)$ 1-converges to $y \in S$ iff there is $\gamma \in D$ and nets $(L ; D, \geqq)$ and $(U ; D, \geqq)$ in $S$ such that

(1a) if $\alpha \geqq \beta \geqq \gamma$, then $L(\beta) \leq L(\alpha) \leq f(\alpha) \leq U(\alpha) \leq U(\beta)$,

(1b) $y=\sup \{L(\alpha): \alpha \geqq \gamma\}=\inf \{U(\alpha): \alpha \geqq \gamma\}$.

Definition 2 . A net $(f ; D, \geqq)$ in a poset $(S, \leq)$ 2-converges to $y \in S$ iff there are nonvoid subsets $M$ and $N$ of $S$ such that

(2a) $M$ is up-directed and $N$ is down-directed,

(2b) $y=\sup M=\inf N$,

(2c) if $m \in M$ and $n \in N$, then there is $\beta \in D$ such that $m \leq f(\alpha) \leq n$ for all $\alpha \geqq \beta$.

Definition 3. A net $(f ; D, \geqq)$ in a poset $(S, \leq)$ 3-converges to $y \in S$ iff there are nonvoid subsets $M$ and $N$ of $S$ such that

(3a) $y=\sup M=\inf N$,

(3b) if $m \in M$ and $n \in N$, then there is $\beta \in D$ such that $m \leq f(\alpha) \leq n$ for all $\alpha \geqq \beta$.

It is easy to see that if a net 1-converges to $y \in S$, then it 2-converges to $y$. Similarly, 2 -convergence implies 3 -convergence. Wolk

Received by the editors May 5, 1966. 
gives an example that shows that 3-convergence need not imply 2convergence and, hence, 3 -convergence need not imply 1-convergence. In $\$ 3$ we give an example that shows that 2 -convergence need not imply 1-convergence and, hence, 3 -convergence need not imply 1 convergence. In $\S 4$ we give a sufficient condition on the poset $(S, \leq)$ that 1 - and 2-convergence be equivalent.

The genealogy of these three modes of convergence is not completely clear. 1-convergence in a poset seems to have been first formulated by Birkhoff $[1$, p. 32]. It is in teresting to note that he does not repeat this definition as such in the second edition $[2$, p. 60]; rather he defines a variant of 1 -convergence in a poset by first embedding the poset in a complete lattice and therein applying the usual generalizations of the notions of limit inferior and limit superior of a sequence. 2-convergence is defined in McShane [6] and seems to be due to him. The major innovation here is that the upper and lower "pinching" of the given net is done in terms of the poset alone, rather than in terms of two other nets, each of which is defined on the same directed set as the given net. It is this lack of comparability between the domain of the net and the up- and down-directed sets $M$ and $N$ that prevents 1 - and 2-convergence from being equivalent. 3 -convergence in the form given in Definition 3, is due to Wolk, who gives references to Rennie [7] and Ward [8]. The latter reformulates 3 -convergence in terms of filters, referring the reader to Rennie for the notion of 3-convergence in terms of nets. In turn, Rennie gives references to Birkhoff [2] and Löwig [5]. An examination of these papers seems to indicate that while the germ of the notion of 3convergence might be said to be in Birkhoff, it is in Löwig that this mode of convergence was first given in something like its present form.

3. Example. Let $\omega$ be the first nonfinite ordinal and $\Omega$ the first noncountable ordinal. Denote by $\omega^{\prime}$ the set of all ordinals not exceeding $\omega$ and by $\Omega^{\prime}$ the set of all ordinals not exceeding $\Omega$. We let $S=\omega^{\prime} \times \Omega^{\prime}$ and think of $S$ as in the figure.

We define a partial order $\leq$ on $S$ as follows:

(1) If $(a, b) \neq(\omega, \Omega)$, then $(a, b)<(\omega, \Omega)$,

(2) $(a, b)<(a, c)$ iff $b<c$,

(3) If $a \neq \omega, b \neq \omega$, and $a \neq b$, then $(a, c)<(b, d)$ iff $a<b$ and $c<d$,

(4) If $a \neq \omega, b \neq \Omega$, and $c \neq \Omega$, then $(a, b)<(\omega, c)$ iff there is an ordinal $t$ having no immediate predecessor such that $b<t \leq c$.

Letting $Z$ denote the positive integers, we define the net $(f ; Z, \geqq)$ in $S$, where $\geqq$ is the usual order for $Z$, by $f(n)=(n, \Omega)$, for $n \in Z$. Letting $M=\{(a, b) \in S: a \neq \omega, b \neq \Omega\}$ and $N=\{(\omega, \Omega)\}$, it is easily 


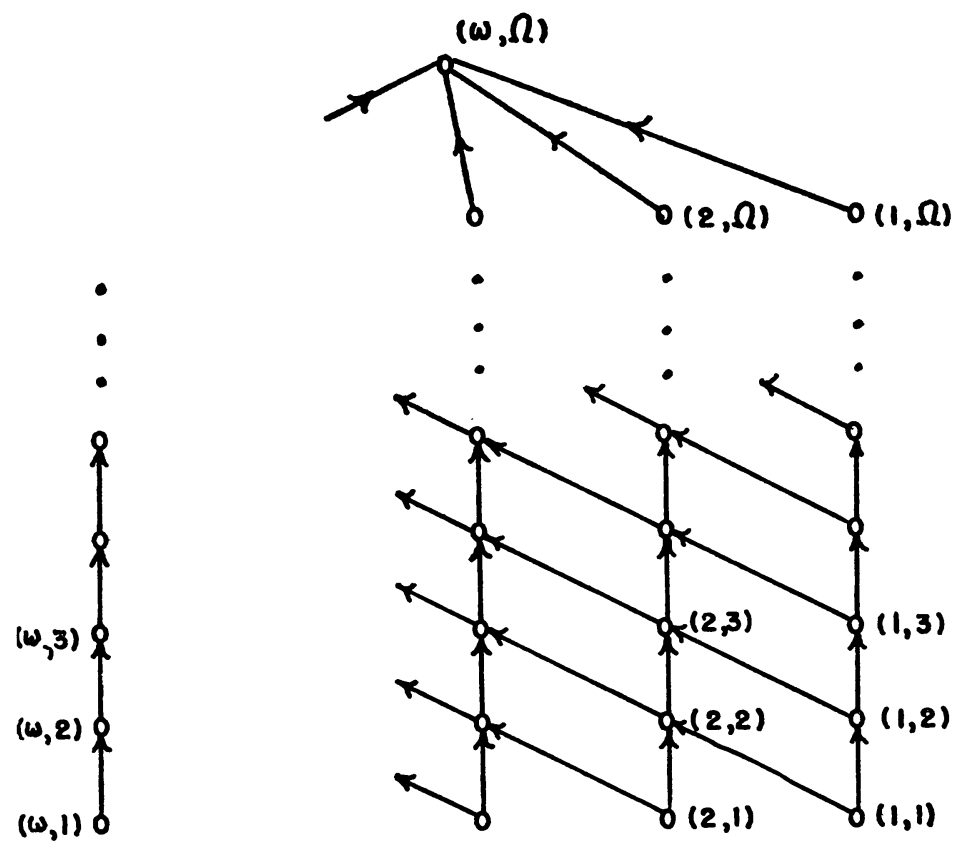

checked that $M$ is up-directed, $N$ is down-directed, and, letting $y=(\omega, \Omega)$, that $\sup M=\inf N=y$. Since $M, N$, and $(f ; Z, \geqq)$ also satisfy (2c) of Definition 2 , it follows that $(f ; Z, \geqq) 2$-converges to $y$.

If we assume that $(f ; Z, \geqq) 1$-converges to $y$, referring to Definition 1 and the figure, it follows from (1a) and the fact that the elements of the range of $f$ are not mutually comparable, that $L_{\gamma}=\{L(\alpha)$ : $\alpha \geqq \gamma\} \subset M$. Since $L_{\gamma}$ is countable it is clear that the statement sup $L_{\gamma}$ $=y$ cannot hold. Thus $(f ; Z$, $\geqq)$ does not 1 -converge to $y$.

4. Sufficient conditions for equivalence. In this section we give a sufficient condition on the poset $(S, \leq)$ that 2 -convergence be equivalent to 1-convergence. One such condition (in fact, all three types of convergence are equivalent under this condition) was noted by Rennie, namely that $(S, \leq)$ be a conditionally complete lattice. We have approached the problem by imposing a certain kind of countability assumption on the ordering of the poset. Our attempts to obtain a condition similar to that which Wolk imposed in order to obtain a near-sufficient condition that 2 - and 3 -convergence be equivalent have been unsuccessful without the imposition of some sort of countability assumption at one place or another.

Definition 4. A poset $(S, \leq)$ is said to have property $B$ iff for each nonvoid subset $J$ of $S$ and $y \in S$ 
(4a) if $J$ is up-directed and $y=\sup J$, there is a countable updirected subset $C$ of $J$ such that $\sup C=y$

(4b) if $J$ is down-directed and $y=\inf J$, there is a countable downdirected subset $C$ of $J$ such that inf $C=y$.

Theorem. Let $(S, \leq)$ be a poset with property B. Let $(f ; D, \geqq)$ be a net in $S$; then $(f ; D, \geqq) 1$-converges to $y \in S$ iff it 2-converges to $y \in S$.

Proof. Since 1-convergence always implies 2-convergence we need only verify the converse. By property $B$ there are countable subsets $M^{\prime}$ and $N^{\prime}$ of $M$ and $N$, respectively, such that $\sup M^{\prime}=\sup M=y$ $=\inf N=\inf N^{\prime}$. Since $M^{\prime}$ and $N^{\prime}$ are up- and down-directed, respectively, there is no loss of generality in assuming that, letting $M^{\prime}$ $=\left\{m_{1}, m_{2}, \cdots\right\}$ and $N^{\prime}=\left\{n_{1}, n_{2}, \cdots\right\}, m_{1} \leq m_{2} \leq \cdots$, and $n_{1} \geq n_{2} \geq \cdots$. Let $X_{i}=\left[m_{i}, n_{i}\right](i=1,2, \cdots)$ and note that $X_{1}$ $\supset X_{2} \supset \cdots$. For each $\alpha \in D$, denote $\{f(\beta): \beta \in D, \beta \geqq \alpha\}$ by $T_{\alpha}$. We begin by choosing $\alpha_{1} \in D$ such that $T_{\alpha_{1}} \subset X_{1}$. Suppose we have chosen $\alpha_{1}, \alpha_{2}, \cdots, \alpha_{n}$ such that $T_{\alpha_{i}} \subset X_{i}, \alpha_{1} \leqq \alpha_{2} \leqq \cdots \leqq \alpha_{n}$, and $\alpha_{i} \geqq \alpha_{j}$ for $1 \leq i<j \leq n$. We then try to choose $\alpha_{n+1}$. Now, there is $\beta^{\prime} \in D$ such that $T_{\beta^{\prime}} \subset X_{n+1}$ and $\beta^{\prime} \geqq \alpha_{n}$. If for each $\beta \geqq \beta^{\prime}$ there is an $i, 1 \leq i \leq n$, such that $\beta \leqq \alpha_{i}$, then $\alpha_{i} \geqq \beta \geqq \beta^{\prime}$ and $T_{\beta^{\prime}} \supset T_{\beta} \supset T_{\alpha_{i}}$. Also, $\alpha_{i} \leqq \alpha_{n} \leqq \beta^{\prime}$ and so $T_{\alpha_{i}} \supset T_{\beta^{\prime}}$. Hence $T_{\beta^{\prime}}=T_{\beta}$ for all $\beta \geqq \beta^{\prime}$. If this is the case, then for each $m \in M$ and $n \in N, T_{\beta^{\prime}} \subset[m, n]$. So if $x \in T_{\beta^{\prime}}, x \in[m, n]$. It follows that $\boldsymbol{x}=\boldsymbol{y}$. Hence $(f ; D, \geqq)$ is eventually constant and we can easily find nets $(U ; D, \geqq)$ and $(L ; D, \geqq)$ satisfying the appropriate conditions. Thus we can assume that $\beta \geqq \beta^{\prime}$ can be found such that $\beta \leqq \alpha_{i}(i=1,2, \cdots, n)$. Let $\alpha_{n+1}=\beta$. We have $T_{\alpha_{n+1}} \subset X_{n+1}, \alpha_{1} \leqq \alpha_{2}$ $\leqq \cdots \leqq \alpha_{n} \leqq \alpha_{n+1}$ and $\alpha_{i} \leqq \alpha_{j}$ for $1 \leq i<j \leq n+1$. It is thus clear that we may assume that there are given $\alpha_{k} \in D(k=1,2, \cdots)$ such that $\alpha_{1} \leqq \alpha_{2} \leqq \cdots, \alpha_{i} \geqq \alpha_{j}$ for $1 \leq i<j$, and $T_{\alpha_{k}} \subset X_{k}$.

It is convenient to denote $m_{i}$ by $l_{\alpha_{i}}$ and $n_{i}$ by $u_{\alpha_{i}}(i=1,2, \cdots)$. Of course $l_{\alpha_{i}} \leq l_{\alpha_{i+1}} \leq y \leq u_{\alpha_{i+1}} \leq u_{\alpha_{i}}$.

We now define nets $(L ; D, \geqq)$ and $(U ; D, \geqq)$. Let $\gamma=\alpha_{1}$ and define $L\left(\alpha_{i}\right)=l_{\alpha_{i}}$ and $U\left(\alpha_{i}\right)=u_{\alpha_{i}}$. If $\alpha \geq \gamma$, define $L(\alpha)=l_{\gamma}$ and $U(\alpha)=u_{\gamma}$. If $\alpha \geqq \gamma$ we proceed as follows: If $\alpha \geqq \alpha_{i}$ for all $i$, define $L(\alpha)=U(\alpha)=y$. If it is false that $\alpha \geqq \alpha_{i}$ for all $i$, then there is a least $j$ such that $\alpha \geqq \alpha_{j}$. Note that $j>1$ and $\alpha \geqq \alpha_{k}$ for $k=j, j+1, \cdots$; also, either $\alpha \leqq \alpha_{j}$ or they are not comparable. Define $L(\alpha)=l_{\alpha_{j-1}}$ and $U(\alpha)=u_{\alpha_{j-1} \text {. }}$.

Now suppose that $\beta \geqq \alpha \geqq \gamma$. If $\alpha=\alpha_{1}=\gamma$, then $L(\alpha) \leq L(\beta)$ since $L(\beta)=l_{\alpha_{i}}$ for some $i$ or $L(\beta)=y$. Next, suppose that $\alpha=\alpha_{i}$ for some $i>1$. If $\beta=\alpha_{j}$ for some $j$, then $L(\alpha) \leq L(\beta)$ since $\beta \geqq \alpha$. If $\beta \neq \alpha_{j}$ for all $j$, then either $\beta \geqq \alpha_{k}$ for all $k$ or there is a least $k$ such that $\beta \geq \alpha_{k}$. In the former case, $L(\alpha) \leq L(\beta)=y$. In the latter case, $l_{\alpha_{i}}=L(\alpha)$ 
$\leq L(\beta)=l_{\alpha_{k-1}}$ since $k-1 \geq i$. We now suppose that $\alpha \neq \alpha_{i}$ for all $i$. If $\alpha \geqq \alpha_{j}$ for all $j$, then $L(\alpha) \leq L(\beta)=y$. Otherwise, there is a least $j$ such that $\alpha \geqq \alpha_{j}$ and $j>1$. Since $\beta \geqq \alpha$, if $\beta \geqq \alpha_{i}$ for all $i$, then $L(\alpha)=l_{\alpha_{i-1}}$ $\leq L(\beta)=y$. Otherwise, there is a least $k$ such that $\beta \geq \alpha_{k}$. Now $k \geq j$ since if $k<j$ then $\beta \geqq \alpha \geqq \alpha_{j-1} \geqq \alpha_{k}$ and this is contradictory. Hence $L(\alpha)=l_{\alpha_{j-1}} \leq l_{\alpha_{k-1}}=L(\beta)$. Thus we have shown that if $\beta \geqq \alpha \geqq \gamma$, then $L(\alpha) \leq L(\beta)$. Similarly, $U(\beta) \leq U(\alpha)$.

Next, if $\beta \geqq \gamma$ and $\beta=\alpha_{i}$ for some $i$, then $l_{\alpha_{i}}=L(\beta) \leq f(\beta) \in T_{\alpha_{i}} \subset X_{i}$. If $\beta \neq \alpha_{i}$ for all $i$ and it is false that $\beta \geqq \alpha_{i}$ for all $i$, then there is a least $j$ such that $\beta \geqq \alpha_{j}$. Thus $\beta \geqq \alpha_{j-1}$ and $L(\beta)=l_{\alpha_{j-1}}$. Since $X_{j-1} \supset T_{\alpha_{j-1}}$ we have $L(\beta) \leq f(\beta)$. If $\beta \geqq \alpha_{i}$ for all $i$, then $L(\beta)=y$. Also $T_{\beta} \subset T_{\alpha_{i}}$ for all $i$ and so $T_{\beta} \subset \cap_{i=1}^{\infty} T_{\alpha_{i}} \subset \cap_{i=1}^{\infty} X_{i}=\{y\}$. Since $T_{\beta} \neq \varnothing$ we have $T_{\beta}=\{y\}$. Hence $f(\beta)=y$ and so $L(\beta) \leq f(\beta)$. Similarly, $f(\beta) \leq U(\beta)$.

We now have: If $\beta \geqq \alpha \geqq \gamma$, then

$$
L(\alpha) \leq L(\beta) \leq f(\beta) \leq U(\beta) \leq U(\alpha) .
$$

We conclude the proof by observing that $\sup \{L(\alpha): \alpha \geqq \gamma\}$ $=\inf \{U(\alpha): \alpha \geqq \gamma\}=y$.

We make two last remarks: (1) Wolks "Property A" and our own property $B$ are somewhat related constraints on a poset. At the same time, there are examples showing that neither implies the other. (2) Property B does not imply that all three types of order convergence considered here are equivalent.

\section{REFERENCES}

1. G. Birkhoff, Lattice theory, Amer. Math. Soc. Colloq. Publ., Vol. 25, Amer. Math. Soc., Providence, R. I., 1940.

2. - Lattice theory, rev. ed., Amer. Math. Soc. Colloq. Publ., Vol. 25, Amer. Math. Soc., Providence, R. I., 1961.

3. O. Frink, Topology in lattices, Trans. Amer. Math. Soc. 51 (1942), 569-582.

4. J. L. Kelley, General topology, Van Nostrand, New York, 1955.

5. $\mathrm{H}$. Löwig, Intrinsic topology and completion of Boolean rings, Ann. of Math. 42 (1941), 1138-1196.

6. E. J. McShane, Order-preserving maps and integration processes, Annals of Mathematics Studies, No. 31, Princeton Univ. Press, Princeton, N. J., 1953.

7. B. C. Rennie, Lattices, Proc. London Math. Soc. (2) 52 (1951), 386-400.

8. A. J. Ward, On relations between certain intrinsic topologies in partially ordered sets, Proc. Cambridge Philos. Soc. 51 (1955), 254-261.

9. E. S. Wolk, On order-convergence, Proc. Amer. Math. Soc 12 (1961), 379-384.

Iowa State University 